\title{
Dyssynchronization reduces dynamic obstruction without affecting systolic function in patients with hypertrophic obstructive cardiomyopathy: a pilot study.
}

Geneviève Giraldeau ${ }^{1,2}$, Nicolas Duchateau ${ }^{1,2,3}$, Bart Bijnens ${ }^{3,4}$, Luigi Gabrielli ${ }^{1,2,5}$, Diego Penela ${ }^{1,2}$, Reinder Evertz $^{1,2}$, Lluis Mont ${ }^{1,2}$, Josep Brugada ${ }^{1,2}$, Antonio Berruezo ${ }^{1,2}$, Marta Sitges ${ }^{1,2, *}$.

Affiliations:

${ }^{1}$ Hospital Clínic, Universitat de Barcelona, c/ Villarroel 170, E-08036 Barcelona, Spain;

${ }^{2}$ Institut d'investigacions Biomèdiques August Pi i Sunyer, c/ Rosselló 149-153, E-08036 Barcelona, Spain;

${ }^{3}$ Universitat Pompeu Fabra, c/ Roc Boronat 138, E-08018 Barcelona, Spain;

${ }^{4}$ Institució Catalana de Recerca i Estudis Avançats, Pg/ Lluís Companys 23, E-08010 Barcelona, Spain.

${ }_{5}^{5}$ División de Enfermedades Cardiovasculares, Pontificia Universidad Católica de Chile, Marcoleta 367, Santiago 22354 3000, Chile;

\section{"Corresponding author:}

Marta Sitges

Cardiology Department, Hospital Clínic,

Calle Villarroel 170, E-08036 Barcelona, Spain

Tel: +34.93.227.5551; Fax +34.93.451.3045

Email: msitges@clinic.ub.es

Word count (excluding tables):

3075 words

Number of tables:

3

Number of figures:

4

\section{Acknowledgements / funding}

The authors acknowledge the Spanish Industrial and Technological Development Center (cvRemod-CEN-20091044), the Subprograma de Proyectos de Investigación en Salud, Instituto de Salud Carlos III, Spain (FIS-PI1 1/01709), the Plan Nacional I+D+I (DEP2010-20565), the Comisión Nacional de Ciencia y Tecnología (FONDAP-15130011), and the European Union 7th Framework Programme (VP2HF-FP7-2013-611823). GG was awarded the grant Bal Du Coeur from the Fondation de l'Institut de Cardiologie de Montréal.

Disclosures / conflict of interest: none declared. 


\section{Abstract}

Purpose: Dyssynchrony from biventricular pacing $(\mathrm{BiV})$ can reduce dynamic obstruction in hypertrophic obstructive cardiomyopathy (HOCM), but its consequences on the left ventricular (LV) systolic function are unknown. We evaluate changes in LV systolic function and assess the effectiveness of $\mathrm{BiV}$ in HOCM.

Methods: 13 patients with HOCM (55[33/75] years, 5 males) received a BiV device and underwent 2D transthoracic echocardiography before the implantation and at 12 months follow-up. Global longitudinal and radial strain, and the timing of segmental displacement curves were measured by commercial speckle-tracking software to assess LV systolic function and dyssynchrony.

Results: Peak gradient in the LV outflow tract (LVOT) significantly decreased from 80[51/100] to $30[5 / 66] \mathrm{mmHg}(\mathrm{p}=0.005)$. LV global strain was preserved from baseline to follow-up: $35.1[20.2 / 43.8] \%$ vs. $32.6[27.1 / 44.1] \%, \quad \mathrm{p}=\mathrm{NS}$ (radial), and $-16.6[-19.1 /-14.4] \%$ vs. $-15.7[-17.0 /-14.2] \%$, p=NS (longitudinal). Dyssynchrony analysis using displacement curves showed inversion of wall motion timing with earlier displacement of the lateral wall at follow-up only in patients with reduction in LVOT gradient.

Conclusions: BiV reduces LVOT obstruction in patients with HOCM when dyssynchronization of LV motion and inversion of the timing of LV wall activation are reached. Notably, this does not lead to further deterioration of LV systolic function at mid-term follow-up.

\section{Keywords}

Speckle-tracking; Myocardial strain; Biventricular pacing; Hypertrophic obstructive cardiomyopathy. 


\section{Abbreviations}

AV: atrio-ventricular

BiV: Biventricular pacing

HOCM: Hypertrophic obstructive cardiomyopathy

LV: Left ventricle

LVOT: Left ventricle outflow tract

RV: Right ventricle 


\section{Introduction}

Sixty percent of patients with hypertrophic cardiomyopathy have significant $(\geq 30 \mathrm{mmHg}$ ) left ventricular outflow tract (LVOT) obstruction, either at rest or after provocation (e.g. a Valsalva maneuver), which contributes to symptoms and increases the risk of sudden cardiac death [1]. Dualchamber pacing is an alternative to surgical therapy or alcohol septal ablation [1].

However, controversial results for dual-chamber pacing efficacy [2] led to the research of alternative modes of pacing. Moreover, recent long-term follow-up data has raised concerns about chronic dual-chamber pacing in HOCM patients and a possible deleterious effect on survival and heart failure [3]. In accordance with this finding, some studies suggested that right ventricular (RV) pacing deteriorates left ventricular function in patients with HOCM both acutely and on the long-term run [4-6]. Similarly, studies in the population with normal or reduced ventricular function and without hypertrophic cardiomyopathy have showed an association between RV-pacing and the deterioration of left ventricle (LV) ejection fraction and the augmentation of the risk of hospitalization for heart failure [7-12].

As an alternative, biventricular pacing (BiV) to reduce dynamic obstruction in hypertrophic obstructive cardiomyopathy (HOCM) has yielded encouraging results for reducing intraventricular obstruction and for clinical improvement [13-21]. However, while its effectiveness was demonstrated, the long-term effect of BiV-pacing on LV function among patients with HOCM and preserved LV ejection fraction is unknown.

The main contribution of this study consists in investigating the consequences of BiV on LV deformation, to determine whether the benefits of pacing-induced dyssynchrony are not hindered by lower LV systolic function. We previously reported on the effects of BiV in terms of LVOT pressure gradient and LV motion on 9 patients with HOCM [13]. The present study extends this work by a more comprehensive assessment of LV systolic function with deformation imaging at mid-term follow-up. 


\section{Methods}

\section{Patients}

Between November 2005 and January 2013, 18 consecutive HOCM patients with significant LVOT obstruction (baseline intraventricular gradient $50 \mathrm{mmHg}$ ), LV ejection fraction $\geq 50 \%$, sinus rhythm and severe symptoms despite optimum medical treatment and without indication for pacing due to bradycardia, were referred for BiV. Patients were accepted in the study only if surgical myomectomy or septal ablation were contraindicated (due to severe renal insufficiency, chronic obstructive pulmonary disease, liver cirrhosis) or were refused by the patient. Our institution's Ethics committee approved the study protocol, and written informed consent was obtained from all patients. Patients were defined as responders to $\mathrm{BiV}$ if they completed both of the following criteria for reduction of obstructive gradient: (i) follow-up gradient $\leq 50 \mathrm{mmHg}$ and (ii) reduction in LVOT pressure gradient of at least $50 \%$ from baseline.

\section{Cardiac resynchronization therapy device implantation}

The technique for device implantation has been described in detail elsewhere [13]. In brief, right leads were positioned conventionally in the right atrial appendage and RV apex. The LV lead was inserted via the coronary sinus and subsequently inserted in a ventricular vein and positioned as laterally as possible on the LV free-wall. All leads were connected to a dual-chamber biventricular implantable device and patients considered being at high risk for sudden cardiac death also received a cardioverterdefibrillator.

\section{Echocardiographic evaluation}

All patients underwent standard transthoracic two-dimensional echocardiography before implantation, one day after implantation (for programming optimization) and follow-up. Parasternal and apical echocardiographic images were obtained with a commercially available system (Vivid7 and E9, GE Healthcare, Milwaukee, WI) using a 3.5-MHz transducer.

LV wall thickness and diameters were measured from the parasternal long-axis view using Mmode or 2D measurements at mitral valve leaflet tips level. LV mass was calculated using the Devereux 
formula [22]. LV volumes and ejection fraction were obtained using the biplane Simpson method. The peak LVOT pressure gradient was determined using continuous wave Doppler from the apical fivechamber view and was measured under resting conditions and after a provocative Valsalva maneuver. Values were determined by averaging measurements obtained in three consecutive cardiac cycles.

Analysis of LV global radial and longitudinal strain was performed from the short-axis view at the papillary muscles level (average of $6 \mathrm{LV}$ segments) and from 4-chamber apical (average of $6 \mathrm{LV}$ segments) view, respectively, using commercial software (2D strain, Echopac, GE Healthcare). We have chosen to analyze GLS only in the 4-chamber as it allows visualizing the septum and the anterolateral wall in the same view, which is of interest to properly assess the changes induced by pacing. Besides, the reliable image quality of this view for strain quantification was also a criterion for privileging it while keeping measurements as simple as possible. The timing of displacement of opposing LV walls was assessed in the short-axis view at the papillary muscles level using displacement curves obtained by speckle-tracking. This view was chosen to explore the mechanisms gradient reduction as the dynamic obstruction predominates at this level. The sample of tissue interrogation was placed in the antero-septal and infero-lateral LV segments. In order to highlight the timing of wall motion, we measured the time-toonset of radial displacement (from beginning of QRS to beginning of wall displacement) and time-topeak of radial displacement (from beginning of QRS to peak systolic displacement). Comparison of timings between baseline and follow-up was made comparing infero-lateral to antero-septal (negative time value signifying that infero-lateral wall motion occurs first, and positive time value signifying that antero-septal displaces first).

\section{Optimization of the programming of the $\mathrm{BiV}$ device}

The algorithm for the optimization of the programming has been previously described elsewhere [13]. The optimum AV interval was considered as the shortest AV interval without A-wave truncation on the mitral inflow. Subsequently, ventricular pacing mode was selected testing RV-only pacing, LV-only pacing, or BiV-pacing with 4 different programs (RV preactivation VV+30ms, simultaneous BiV-pacing VV0ms, or LV preactivation VV-30ms, -60ms). The final pacing configuration was selected according to the maximum reduction of LVOT gradient after a $5 \mathrm{~min}$ equilibrium phase for each setup. The echocardiographer was blinded to pacing configuration. 


\section{Statistical analysis}

Due to the sample size of the study population, non-parametric test were used. Quantitative variables were expressed as median and first/third inter-quartile range while qualitative variables were described as number of cases (\% from reference population). Paired dichotomous and quantitative variables were compared using Chi-square and Wilcoxon tests, respectively. A p-value $<0.05$ was considered statistically significant.

\section{Results}

Of the 18 patients, 1 did not complete the mid-term follow-up at the moment of analysis, and 4 patients did not have images available for strain analysis, leaving a final cohort study of 13 patients with complete echocardiographic follow-up. Baseline characteristics are depicted in Table 1. Follow-up examination was performed at 12 months after implantation. Among the 13 patients in the study, optimum programming of the device based on maximum reduction in the LVOT gradient was VV+30 in 1 patient (8\%), VV0 in 3 patients (23\%), VV-30 in 7 patients $(54 \%), \mathrm{VV}-60$ in 1 patient (8\%) and LV (activation of LV only) in 1 patient (8\%).

\section{LVOT Obstruction}

Median peak LVOT gradient was $80[51 / 100] \mathrm{mmHg}$ at baseline and significantly diminished after the activation of the resynchronization device to $45[20 / 60] \mathrm{mmHg}$ and 30 [5/66] $\mathrm{mmHg}$ at follow-up $(\mathrm{p}=0.005)$. Dynamic gradient with Valsalva maneuver was $110[78 / 130] \mathrm{mmHg}$ before $\mathrm{BiV}$ and significantly diminished to $54[8 / 75] \mathrm{mmHg}$ at follow-up $(\mathrm{p}=0.008)$.

According to the reduction of LVOT obstruction, 8 (62\%) patients were classified as responders and $5(38 \%)$ patients as non-responders to BiV. Individual behaviors are shown in Figure 1.

\section{Intra- and inter-observer variability}

Repeatability in the extraction of myocardial motion, as conditioned by the speckle-tracking procedure, was previously reported [23]. These results are complemented by the measurements 
summarized in Figure 2, which depict repeated curves along the whole cycle at basal septal/lateral levels and global values. Both indicate the low variability in the speckle-tracking measurements.

\section{Impact of $\mathrm{BiV}$ on $\mathrm{LV}$ function}

There was no significant change in the LV function according to ejection fraction estimated by the Simpson method. LV ejection fraction was $71[67 / 73] \%$ at baseline and $69[65 / 75] \%$ at follow-up $(\mathrm{p}=\mathrm{NS})$. Similarly, the LV systolic function as assessed by strain analysis did not significantly changed at follow-up. Global LV radial strain was 35.1[20.2/43.8] \% at baseline and 32.6[27.2/44.1] \% at follow-up $(\mathrm{p}=\mathrm{NS})$. Global longitudinal LV strain was $-16.6[-19.1 /-14.4] \%$ at baseline and $-15.70[-17.0 /-14.2] \%$ at follow-up ( $\mathrm{p}=\mathrm{NS}$ ). (Table 2) Segmental LV strain analysis (radial and longitudinal) was not significantly different between baseline and follow-up, with the exception of the longitudinal strain of the septal-apical segment (-23.8[-34.3/-22.6] \% to $-21.9[-24.4 /-19.0] \%, \mathrm{p}=0.009)$. (Figure 3) Finally, reductions in septal and posterior wall thickness were observed without significant changes in LV cavity size after BiV. (Table 1) This trend was mainly observed in patients who reduced LVOT gradient (responders) as shown in Table 2.

\section{Impact of BiV on LV dyssynchrony}

The changes in displacement of opposing LV walls from baseline to follow-up were significantly different between responders and non-responders to BiV (Table 3). In the responders, we observed a significant delay of the onset of antero-septal displacement $(30[20 / 42] \mathrm{ms}$ to $123[102 / 174] \mathrm{ms}, \mathrm{p}=0.028)$ combined with a non-significant delay of the onset of infero-lateral displacement $(95[25 / 127] \mathrm{ms}$ to $127[103 / 138] \mathrm{ms})$ with BiV resulting in a significant $(\mathrm{p}=0.046)$ inversion in the timing of wall motion as compared to baseline: onset changing from antero-septal first to infero-lateral first.

In the responders, we also observed a significant delay of the time to antero-septal peak displacement $(363[320 / 407] \mathrm{ms}$ to $542[481 / 577] \mathrm{ms})(\mathrm{p}=0.028)$, combined with a non-significant delay of the time to infero-lateral peak displacement $(421[358 / 515] \mathrm{ms}$ to $418[367 / 455] \mathrm{ms})$ resulting in a significant $(\mathrm{p}=0.028)$ inversion in the timing of peak wall displacement as compared to baseline, changing from antero-septal wall first (infero-lateral 29[0/147] ms after antero-septal peak) to inferolateral wall peak first (infero-lateral -108[-186/-62] ms before antero-septal peak). 
The inversion of the timing of wall displacement (onset and peak) was absent in the nonresponders. Representative examples of responder vs non-responder patients are illustrated in Figure 4.

\section{Discussion}

In this study on patients with HOCM at mid-term follow-up, we demonstrate that (i) BiV does not affect LV systolic function, and that (ii) LVOT obstruction is reduced when dyssynchronization of LV motion and inversion of the timing of LV wall activation are reached. This reduction of dynamic obstruction is associated with a change in the timings of the motion of opposing LV walls.

\section{Biventricular pacing in $\mathrm{HOCM}$}

Current guidelines include cardiac pacing in HOCM as a class IIb indication and recommend dualchamber pacing in patients without bradycardia indication who are symptomatic despite optimum medical treatment and who are suboptimal candidates for invasive septal reduction (surgical or alcohol reduction) [1]. Although, the initials results for dual-chamber pacing were positive, Nishimura et al. showed in a randomized trial a weak reduction of LVOT gradient $(<50 \%)$ and no improvement in exercise capacity on a short-term follow-up, suggesting a placebo effect for the observed symptomatic improvement [2]. More recently, $\mathrm{BiV}$ has shown to induce a significant gradient reduction from $74 \pm 23$ $\mathrm{mmHg}$ to $28 \pm 17 \mathrm{mmHg}$ in 9 patients at 1-year follow-up in HOCM patients [13]. Lenarczyk et al. found similar results demonstrating significant reduction of peak gradient ( $60 \%$ reduction) in 9 patients with HOCM treated with BiV [15]. Similarly, smaller studies and case reports have shown the efficacy of BiV or LV-pacing for gradient reduction in the HOCM population [14,16-21]. Our results confirm a significant reduction of peak LVOT gradient at follow-up on a larger population (from 80 [51/100] $\mathrm{mmHg}$ to $30[5 / 66] \mathrm{mmHg}, \mathrm{p}=0.005)$.

\section{Dyssynchronization as mechanism for LVOT obstruction relief}

The usual indication for BiV-pacing is for symptomatic patients with low LV ejection fraction ( $\leq$ $35 \%$ ) with QRS prolongation and is aimed at resynchronizing electromechanical activity of the heart with 
the goal of improving ventricular function [24]. It is important to emphasize that the objective of BiVpacing in patients with HOCM is different, as it is indeed intended to dyssynchronize ventricular contraction and change the pattern of mechanical activation in order to reduce the dynamic obstruction. Using wall displacement traces (antero-septal and infero-lateral segments), we observed the inversion of the order of wall displacement, only in the responders, suggesting that the induced mechanical dyssynchrony plays a role in the reduction of LVOT obstruction.

\section{Impact of BiV-pacing on LV function}

In our population, we found a global LV longitudinal strain of $-16.6[-19.1 /-14.4] \%$ and radial strain was 35.1[20.2/43.8]\% at baseline, consistent with impaired LV myocardial deformation [25]. These findings are in keeping with previous reports describing reduced myocardial strain despite normal or supranormal LV ejection fraction in HOCM patients [26-28].

Additionally, as shown in previous studies [26-29], regional septal longitudinal deformation is markedly reduced and this does not change after BIV-pacing. To our knowledge, the long-term effect of BiV-pacing on LV function in HCOM is unknown and our data are the first to reveal non-significant changes in LV radial and longitudinal strain or in LV ejection fraction.

\section{RV-pacing in HCOM}

From our results, the induced change in mechanical displacement of the LV free-wall, towards moving before the septum, is crucial for response. Therefore, the actual controversy about efficacy and placebo effect for RV-pacing in HOCM might be explained by the induction of a too small motion change of the interventricular septum, possibly due to the site of pacing being too close to the LV apex. By changing the timing of mechanical LV activation and inverting LV wall displacement, BiV might create changes in the 3-dimensional geometry of the LVOT and mitral valve apparatus resulting in the reduction of obstruction. As previously demonstrated [13], the acute reduction of gradient with BiVpacing supports this mechanical explanation for response and is corroborated by other short-term studies [15-17,19-21,30]. On a long-term basis, LV reverse remodeling might play an additional role as shown by significant reduction of septal and posterior wall thickness. Previous studies showed that in HOCM patients treated with RV-pacing, there was a gradient reduction during follow-up even when pacing was 
turned off, suggesting a role of remodeling on the longer term [31].

\section{Impact of RV-pacing on LV function}

$\mathrm{LV}$ dysfunction as a consequence of chronic RV-pacing is becoming an increasingly recognized entity in patients without HCM. In the PACE trial, deterioration of ejection fraction in the RV-pacing group was observed in comparison to no change of ejection fraction in CRT-pacing group [7,8]. The MOST study showed that cumulative percentage of pacing was a predictor for heart failure hospitalization $[9,10]$. Similarly, Tantengco et al. showed significant LV deterioration at long-term follow-up in 24 patients with congenital AV block in comparison to controls [11]. The DAVID study also showed that RV-pacing might be deleterious and increase heart failure events in some patients with ejection fraction $\leq 40 \%[32]$.

In hypertrophic cardiomyopathy, results are controversial. Lucon et al. reported the very long-term follow-up of 51 patients with HOCM treated with dual-chamber pacing and showed a non-significant reduction of ejection fraction (64\% to 56\%) [4]. Nishimura et al. and Betocchi et al. found LV diastolic and systolic deterioration during acute RV-pacing [5,6]. Moreover, recent long-term follow-up data in HOCM patients showed possible deleterious effect on survival and heart failure of RV-pacing in comparison to other management [3]. Conversely, others have showed no EF deterioration with dualchamber pacing $[31,33]$.

While there is increasing evidence that RV-pacing deteriorates LV function in a lot of clinical settings, BiV-pacing has not been shown to induce the same effect and has rather been suggested as corrective therapy for RV-pacing induced LV dysfunction. This, together with our findings, suggests a benefit for preferring BiV-pacing in the HOCM population.

\section{Limitations}

The main limitation of this study is the small cohort of patients. Low study size might hinder the power of statistical comparisons and in particular the assessment of the lack of differences. Thus, our findings are preliminary data and have to be confirmed by larger studies. Nonetheless, it has to be noted that experience with $\mathrm{BiV}$ in HOCM patients is worldwide limited, and that our study presents a broader 
population than previously published works.

We are aware that choosing a definition for response to classify responders and non-responders is arbitrary but the criteria were chosen taking into consideration what might appear to be clinically relevant. A spectrum of response would be preferable but this was impossible considering the population size.

We are also aware about the limitations of dyssynchrony measures especially when considered as predictors of outcome; however, it was not our principal objective, but rather an exploratory research for the mechanism of action. Moreover, inherent characteristics of HOCM (myocardial fiber disarray, markedly abnormal strain [34,35] and heterogeneous involvement of LV myocardial segment) pose a challenge for motion analysis. Potentially, more precise methods for the quantification of motion changes between baseline and follow-up may allow depicting more comprehensively these changes [23]. Besides, our analysis was only performed at rest. Its extension to stress conditions may be of added value, and should be considered for future studies.

\section{Conclusions}

Our results show that BiV reduces LVOT obstruction in patients with HOCM, by dyssynchronizing LV motion and inverting the timing of LV wall activation. Our study confirms that BiV-pacing is efficient in this task, as this does not lead to further deterioration of LV systolic function at mid-term follow-up. This is clinically reassuring and suggests a benefit for preferring BiV-pacing in the HOCM population. The confirmation of this finding would deserve further prospective larger studies.

\section{References}

1. Gersh BJ, Maron BJ, Bonow RO, Dearani JA, Fifer MA, Link MS, Naidu SS, Nishimura RA, Ommen SR, Rakowski H, Seidman CE, Towbin JA, Udelson JE, Yancy CW; American College of Cardiology Foundation/American Heart Association Task Force on Practice Guidelines (2011) 2011 $\mathrm{ACCF} / \mathrm{AHA}$ guideline for the diagnosis and treatment of hypertrophic cardiomyopathy: a report of the American College of Cardiology Foundation/American Heart Association Task Force on Practice Guidelines. J Am Coll Cardiol 58:e212-260. doi: 10.1016/j.jacc.2011.06.011 
2. Nishimura RA, Trusty JM, Hayes DL, Ilstrup DM, Larson DR, Hayes SN, Allison TG, Tajik AJ (1997) Dual-chamber pacing for hypertrophic cardiomyopathy: a randomized, double-blind, crossover trial. J Am Coll Cardiol 29:435-441. doi: 10.1016/S0735-1097(96)00473-1

3. Ball W, Ivanov J, Rakowski H, Wigle ED, Linghorne M, Ralph-Edwards A, Williams WG, Schwartz L, Guttman A, Woo A (2011) Long-term survival in patients with resting obstructive hypertrophic cardiomyopathy comparison of conservative versus invasive treatment. J Am Coll Cardiol 58:2313-2321. doi: 10.1016/j.jacc.2011.08.040

4. Lucon A, Palud L, Pavin D, Donal E, Behar N, Leclercq C, Mabo P, Daubert JC (2013) Very late effects of dual chamber pacing therapy for obstructive hypertrophic cardiomyopathy. Arch Cardiovasc Dis 106:373-381. doi: 10.1016/j.acvd.2013.04.003

5. Nishimura RA, Hayes DL, Ilstrup DM, Holmes DR Jr, Tajik AJ (1996) Effect of dual-chamber pacing on systolic and diastolic function in patients with hypertrophic cardiomyopathy. Acute Doppler echocardiographic and catheterization hemodynamic study. J Am Coll Cardiol 27:421-430. doi: 10.1016/0735-1097(95)00445-9

6. Betocchi S, Losi MA, Piscione F, Boccalatte M, Pace L, Golino P, Perrone-Filardi P, Briguori C, Franculli F, Pappone C, Salvatore M, Chiariello M (1996) Effects of dual-chamber pacing in hypertrophic cardiomyopathy on left ventricular outflow tract obstruction and on diastolic function. Am J Cardiol 77:498-502. doi: 10.1016/S0002-9149(97)89344-7

7. Dickstein K (2011) Chronic right ventricular pacing, adverse remodelling, and CRT: an ounce of prevention? Eur Heart J 32:2483-2485. doi: 10.1093/eurheartj/ehr337

8. Yu CM, Chan JY, Zhang Q, Omar R, Yip GW, Hussin A, Fang F, Lam KH, Chan HC, Fung JW (2009) Biventricular pacing in patients with bradycardia and normal ejection fraction. N Engl J Med 361:2123-2134. doi: 10.1056/NEJMoa0907555

9. Sweeney MO, Hellkamp AS, Ellenbogen KA, Greenspon AJ, Freedman RA, Lee KL, Lamas GA; MOde Selection Trial Investigators (2003) Adverse effect of ventricular pacing on heart failure and atrial fibrillation among patients with normal baseline QRS duration in a clinical trial of pacemaker therapy for sinus node dysfunction. Circulation 107:2932-2937. doi: 10.1161/01.CIR.0000072769.17295.B1 
10. De Sisti A, Marquez MF, Tonet J, Bonny A, Frank R, Hidden-Lucet F (2012) Adverse effects of long-term right ventricular apical pacing and identification of patients at risk of atrial fibrillation and heart failure. Pacing Clin Electrophysiol 35:1035-1043. doi: 10.1111/j.1540-8159.2012.03371.x

11. Tantengco MV, Thomas RL, Karpawich PP (2001) Left ventricular dysfunction after long-term right ventricular apical pacing in the young. J Am Coll Cardiol 37:2093-2100. doi: 10.1016/S07351097(01)01302-X

12. Manolis AS (2006) The deleterious consequences of right ventricular apical pacing: time to seek alternate site pacing. Pacing Clin Electrophysiol 29:298-315. doi: 10.1111/j.15408159.2006.00338.x

13. Berruezo A, Vatasescu R, Mont L, Sitges M, Perez D, Papiashvili G, Vidal B, Francino A, Fernández-Armenta J, Silva E, Bijnens B, González-Juanatey JR, Brugada J (2011) Biventricular pacing in hypertrophic obstructive cardiomyopathy: a pilot study. Heart Rhythm 8:221-227. doi: 10.1016/j.hrthm.2010.10.010

14. Vatasescu R, Evertz R, Mont L, Sitges M, Brugada J, Berruezo A (2012) Biventricular / left ventricular pacing in hypertrophic obstructive cardiomyopathy: an overview. Indian Pacing Electrophysiol J 12:114-123.

15. Lenarczyk R, Woźniak A, Kowalski O, Sokal A, Pruszkowska-Skrzep P, Sredniawa B, Szulik M, Zielińska T, Kukulski T, Stabryła J, Mazurek M, Białkowski J, Kalarus Z (2011) Effect of cardiac resynchronization on gradient reduction in patients with obstructive hypertrophic cardiomyopathy: preliminary study. Pacing Clinical Electrophysiol 34:1544-1552. doi: 10.1111/j.15408159.2011.03193.x

16. Rinaldi CA, Kirubakaran S, Bucknall CA, Bostock J, Gill JS (2011) Initial experience of a cohort of patients with hypertrophic cardiomyopathy undergoing biventricular pacing. Indian Pacing Electrophysiol J 11:5-14.

17. Lenarczyk R, Kowalski O, Kukulski T, Kowalczyk J, Kalarus Z (2007) Resynchronization or dyssynchronization--successful treatment with biventricular stimulation of a child with obstructive hypertrophic cardiomyopathy without dyssynchrony. J Cardiovasc Electrophysiol 18:542-544. doi: $10.1111 / \mathrm{j} .1540-8167.2007 .00763 . x$

18. Rogers DP, Marazia S, Chow AW, Lambiase PD, Lowe MD, Frenneaux M, McKenna WJ, Elliott PM (2008) Effect of biventricular pacing on symptoms and cardiac remodelling in patients with 
end-stage hypertrophic cardiomyopathy. Eur J Heart Fail 10:507-13. doi: 10.1016/j.ejheart.2008.03.006

19. Yufu K, Takahashi N, Ooie T, Shigematsu S, Hara M, Sako H, Miyamoto S, Hadama T, Tsuchiya T, Honda T, Yoshimatsu H, Saikawa T (2004) Improved hypertrophic obstructive cardiomyopathy by left ventricular apex epicardial pacing. Intern Med 43:295-299. doi: 10.2169/internalmedicine.43.295

20. Komsuoglu B, Vural A, Agacdiken A, Ural D (2006) Effect of biventricular pacing on left ventricular outflow tract pressure gradient in a patient with hypertrophic cardiomyopathy and normal interventricular conduction. J Cardiovasc Electrophysiol 17:207-209. doi: 10.1111/j.15408167.2005.00291.x

21. Rinaldi CA, Bucknall CA, Gill JS (2002) Beneficial effects of biventricular pacing in a patient with hypertrophic cardiomyopathy and intraventricular conduction delay. Heart 87:e6. doi: 10.1136/heart.87.6.e6

22. Lang RM, Bierig M, Devereux RB, Flachskampf FA, Foster E, Pellikka PA, Picard MH, Roman MJ, Seward J, Shanewise JS, Solomon SD, Spencer KT, Sutton MS, Stewart WJ; Chamber Quantification Writing Group; American Society of Echocardiography's Guidelines and Standards Committee; European Association of Echocardiography (2005) Recommendations for chamber quantification: a report from the American Society of Echocardiography's Guidelines and Standards Committee and the Chamber Quantification Writing Group, developed in conjunction with the European Association of Echocardiography, a branch of the European Society of Cardiology. J Am Soc Echocardiogr 18:1440-1463. doi: 10.1016/j.echo.2005.10.005

23. Duchateau N, Giraldeau G, Gabrielli L, Fernández-Armenta J, Penela D, Evertz R, Mont L, Brugada J, Berruezo A, Sitges M, Bijnens BH (2015) Quantification of local changes in myocardial motion by diffeomorphic registration of currents: Application to paced hypertrophic obstructive cardiomyopathy in 2D echocardiographic sequences. Med Image Anal 19:203-219. doi: 10.1016/j.media.2014.10.005

24. Tracy CM, Epstein AE, Darbar D, DiMarco JP, Dunbar SB, Estes NA 3rd, Ferguson TB Jr, Hammill SC, Karasik PE, Link MS, Marine JE, Schoenfeld MH, Shanker AJ, Silka MJ, Stevenson LW, Stevenson WG, Varosy PD, Ellenbogen KA, Freedman RA, Gettes LS, Gillinov AM, Gregoratos G, Hayes DL, Page RL, Stevenson LW, Sweeney MO; American College of Cardiology 
Foundation; American Heart Association Task Force on Practice Guidelines; Heart Rhythm Society (2012) 2012 ACCF/AHA/HRS focused update of the 2008 guidelines for device-based therapy of cardiac rhythm abnormalities: a report of the American College of Cardiology Foundation/American Heart Association Task Force on Practice Guidelines and the Heart Rhythm Society [corrected]. Circulation 126:1784-1800. doi: 10.1161/CIR.0b013e3182618569

25. Yingchoncharoen T, Agarwal S, Popovic ZB, Marwick TH (2013) Normal ranges of left ventricular strain: a meta-analysis. J Am Soc Echocardiogr 26:185-191. doi: 10.1016/j.echo.2012.10.008

26. Cikes M, Sutherland GR, Anderson LJ, Bijnens BH (2010) The role of echocardiographic deformation imaging in hypertrophic myopathies. Nat Rev Cardiol 7:384-396. doi: 10.1038/nrcardio. 2010.56

27. Yang H, Carasso S, Woo A, Jamorski M, Nikonova A, Wigle ED, Rakowski H (2010) Hypertrophy pattern and regional myocardial mechanics are related in septal and apical hypertrophic cardiomyopathy. J Am Soc Echocardiogr 23:1081-1089. doi: 10.1016/j.echo.2010.06.006

28. Serri K, Reant P, Lafitte M, Berhouet M, Le Bouffos V, Roudaut R, Lafitte S (2006) Global and regional myocardial function quantification by two-dimensional strain: application in hypertrophic cardiomyopathy. J Am Coll Cardiol 47:1175-1181. doi: 10.1016/j.jacc.2005.10.061

29. Paraskevaidis IA, Farmakis D, Papadopoulos C, Ikonomidis I, Parissis J, Rigopoulos A, Iliodromitis EK, Kremastinos DT (2009) Two-dimensional strain analysis in patients with hypertrophic cardiomyopathy and normal systolic function: a 12-month follow-up study. Am Heart J 158:444450. doi: 10.1016/j.ahj.2009.06.013

30. Truin G, Backx A, van Wetten H, Neeleman C (2007) Cardiac resynchronization therapy for mitral systolic anterior motion in a child. Ann Thorac Surg 83:1873-1874. doi: 10.1016/j.athoracsur.2006.11.036

31. Fananapazir L, Epstein ND, Curiel RV, Panza JA, Tripodi D, McAreavey D (1994) Long-term results of dual-chamber (DDD) pacing in obstructive hypertrophic cardiomyopathy. Evidence for progressive symptomatic and hemodynamic improvement and reduction of left ventricular hypertrophy. Circulation 90:2731-2742. doi: 10.1161/01.CIR.90.6.2731

32. Wilkoff BL, Cook JR, Epstein AE, Greene HL, Hallstrom AP, Hsia H, Kutalek SP, Sharma A; Dual Chamber and VVI Implantable Defibrillator Trial Investigators (2002) Dual-chamber pacing or 
ventricular backup pacing in patients with an implantable defibrillator: the Dual Chamber and VVI Implantable Defibrillator (DAVID) Trial. JAMA 288:3115-3123. doi: 10.1001/jama.288.24.3115

33. Yue-Cheng H, Zuo-Cheng L, Xi-Ming L, Yuan DZ, Dong-Xia J, Ying-Yi Z, Hui-Ming Y, HongLiang C (2013) Long-term follow-up impact of dual-chamber pacing on patients with hypertrophic obstructive cardiomyopathy. Pacing Clin Electrophysiol 36:86-93. doi: 10.1111/pace

34. Carasso S, Yang H, Woo A, Vannan MA, Jamorski M, Wigle ED, Rakowski H (2008) Systolic myocardial mechanics in hypertrophic cardiomyopathy: novel concepts and implications for clinical status. J Am Soc Echocardiogr 21:675-683. doi: 10.1016/j.echo.2007.10.021

35. Popovic ZB, Kwon DH, Mishra M, Buakhamsri A, Greenberg NL, Thamilarasan M, Flamm SD, Thomas JD, Lever HM, Desai MY (2008) Association between regional ventricular function and myocardial fibrosis in hypertrophic cardiomyopathy assessed by speckle tracking echocardiography and delayed hyperenhancement magnetic resonance imaging. J Am Soc Echocardiogr 21:12991305. doi: 10.1016/j.echo.2008.09.011 
Table 1. Baseline and follow-up characteristics of the studied population $(\mathbf{N}=13)$. Data are expressed as median (1st/3rd quartiles).

\begin{tabular}{|c|c|c|c|}
\hline & Baseline & Follow-up & p-value \\
\hline Sex (female, \%) & \multicolumn{2}{|c|}{$8(62 \%)$} & NS \\
\hline Age (years) & \multicolumn{2}{|c|}{$55(33 / 75)$} & NS \\
\hline Rest LVOT gradient (mmHg) & $80(51 / 100)$ & $30(5 / 66)$ & 0.005 \\
\hline Provoked LVOT gradient (mmHg) & $110(78 / 130)$ & $54(8 / 75)$ & 0.008 \\
\hline \multirow[t]{2}{*}{ NYHA class (patients, $\%$ ) } & $3(23)$ & $10(77)$ & $\mathrm{p}<0.005$ \\
\hline & $10(77)$ & $3(23)$ & $\mathrm{p}<0.005$ \\
\hline Heart Rate (bpm) & $67(58 / 74)$ & $62(55 / 71)$ & NS \\
\hline QRS duration (ms) & $85(79 / 102)$ & $120(113 / 125)$ & 0.011 \\
\hline E/A ratio & $1.6(0.9 / 1.9)$ & $1.3(0.6 / 1.5)$ & $\mathrm{p}<0.005$ \\
\hline LVEDV (ml) & $44(35 / 49)$ & $39(35 / 49)$ & NS \\
\hline LVESV (ml) & $12(10 / 17)$ & $12(9 / 16)$ & NS \\
\hline LVEF (\%) & $71(67 / 73)$ & $69(65 / 75)$ & NS \\
\hline Short-axis radial strain (\%) & $35.1(20.2 / 43.8)$ & $32.6(27.1 / 44.1)$ & NS \\
\hline 4-chamber longitudinal strain (\%) & $-16.6(-19.1 /-14.4)$ & $-15.7(-17.0 /-14.2)$ & NS \\
\hline Septal thickness (mm) & $20(18 / 27)$ & $20(16 / 26)$ & 0.028 \\
\hline Posterior wall thickness (mm) & $12(11 / 13)$ & $11(10 / 12)$ & 0.016 \\
\hline LV mass (g) & $247(203 / 363)$ & $257(171 / 364)$ & NS \\
\hline
\end{tabular}


Table 2: $\mathrm{LV}$ remodeling and systolic function with $\mathrm{BiV}$ in responders and non-responders. Data are expressed as median (1st/3rd quartiles).

\begin{tabular}{|c|c|c|c|c|}
\hline & \multicolumn{2}{|c|}{ Responders $(\mathrm{n}=8)$} & \multicolumn{2}{|c|}{ Non-Responders $(\mathrm{n}=\mathbf{5})$} \\
\hline & Baseline & Follow-up & Baseline & Follow-up \\
\hline Rest LVOT gradient (mmHg) & $96(40 / 111)$ & $15(0 / 30)^{*}$ & $76(64 / 83)$ & $70(55 / 88)$ \\
\hline Provoked LVOT gradient (mmHg) & $111(71 / 149)$ & $33(0 / 56)^{*}$ & $110(85 / 120)$ & $76(72 / 120)$ \\
\hline Heart rate (bpm) & $60(56 / 72)$ & $60(52 / 71)$ & $68(63 / 82)$ & $67(59 / 74)$ \\
\hline LVEDV (ml) & $45(39 / 54)$ & $40(34 / 60)$ & $44(35 / 49)$ & $39(35 / 49)$ \\
\hline LVESV (ml) & $12(10 / 18)$ & $13(11 / 16)$ & $12(10 / 17)$ & $12(9 / 16)$ \\
\hline LVEF (\%) & $71(67 / 74)$ & $69(65 / 75)$ & $69(63 / 72)$ & $71(55 / 74)$ \\
\hline \multirow[t]{2}{*}{$\mathrm{LV}$ radial strain $(\%)$} & 31.4 & 32.1 & 37.3 & 35.5 \\
\hline & $(22.9 / 48.1)$ & $(25.4 / 40.2)$ & $(14.5 / 43.5)$ & $(23.8 / 55.9)$ \\
\hline \multirow[t]{2}{*}{ LV longitudinal strain (\%) } & -17.1 & -15.6 & -14.7 & -13.8 \\
\hline & $(-20.0 /-14.7)$ & $(-17.1 /-14.7)$ & $(-17.8 /-12.5)$ & $(-17.4 /-11.7)$ \\
\hline Septal thickness (mm) & $24(19 / 28)$ & $21(18 / 26)$ & $19(17 / 27)$ & $16(15 / 26)$ \\
\hline LV Posterior wall thickness (mm) & $12(10 / 13)$ & $11(10 / 11) \dagger$ & $12(12 / 14)$ & $12(11 / 14)$ \\
\hline LV mass (g) & $358(215 / 364)$ & $268(203 / 378)$ & $239(187 / 374)$ & $227(136 / 379)$ \\
\hline
\end{tabular}


systolic volume, $\mathrm{LVEF}=$ Left ventricular ejection fraction; $* \mathrm{p}<0.02$ (baseline vs. follow-up); $\uparrow \mathrm{p}<0.05$ (baseline vs. followup). 
Preprint version accepted to appear in International Journal of Cardiovascular Imaging.

Final version of this paper available at http://www.springerlink.com

Table 3: Analysis of mechanical LV dyssynchrony with LV radial displacement in responders and nonresponders to $\mathrm{BiV}$.

\begin{tabular}{|c|c|c|c|c|c|c|}
\hline & & & \multicolumn{2}{|c|}{ Responders $(n=8)$} & \multicolumn{2}{|c|}{ Non-Responders $(n=5)$} \\
\hline & & & Baseline & Follow-up & Baseline & Follow-up \\
\hline \multirow{6}{*}{ 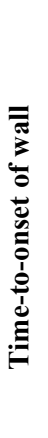 } & \multirow{6}{*}{ 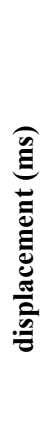 } & $\Delta$ Time-to-onset (IL-AS) & 74 & $-16 \dagger$ & 0 & -19 \\
\hline & & & $(-4 / 86)$ & $(-60 / 31)$ & $(0 / 15)$ & $(-71 /-6)$ \\
\hline & & Septal Onset (ms) & 30 & $123^{*}$ & 30 & $90 \dagger$ \\
\hline & & & $(20 / 42)$ & $(102 / 174)$ & $(21 / 52)$ & $(58 / 118)$ \\
\hline & & Infero-Lateral Onset (ms) & 95 & 127 & 30 & 45 \\
\hline & & & $(25 / 127)$ & $(103 / 138)$ & $(21 / 67)$ & $(28 / 84)$ \\
\hline \multirow{6}{*}{ 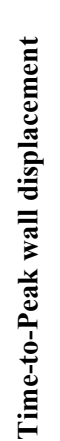 } & \multirow{6}{*}{$\stackrel{\overparen{\mathscr{E}}}{\mathrm{E}}$} & $\Delta$ Time-to-peak (IL-AS) & 29 & $-108^{*}$ & 0 & 0 \\
\hline & & & $(0 / 147)$ & $(-186 /-62)$ & $(-8 / 137)$ & $(-177 / 37)$ \\
\hline & & Septal peak (ms) & 363 & $542 *$ & 363 & 441 \\
\hline & & & $(320 / 407)$ & $(481 / 577)$ & $(285 / 374)$ & $(385 / 488)$ \\
\hline & & Infero-Lateral peak (ms) & 421 & 418 & 368 & 374 \\
\hline & & & $(358 / 515)$ & $(367 / 455)$ & $(364 / 422)$ & $(287 / 479)$ \\
\hline
\end{tabular}

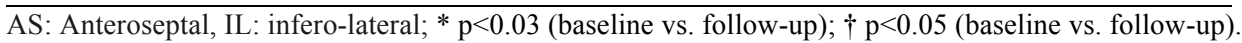




\section{Figures legend}

\section{Figure 1: LVOT pressure gradient evolution}

Figure 2: Repeatability in the extraction of radial displacement and global longitudinal strain, as conditioned by the speckle-tracking procedure. Traces shown for the basal septal and lateral wall locations (radial displacement, left) and the whole myocardium (global longitudinal strain, right). Left: Intra-observer variability (single observer, single cycle, repeated 10 times). Middle: Inter-observer variability (2 different observers, single cycle). Right: Influence of the echocardiographic data (single observer, 3 consecutive cycles and 1 additional cycle from another sequence of the same patient [4-chamber view zoomed-in on the LV])

Figure 3: Global and segmental strain analysis: Baseline to Follow-up. Left: segmental and global radial peak systolic LV strain derived from the short-axis view at baseline (white) and follow-up (black). Right: segmental and global longitudinal peak systolic LV strain derived from the 4-chamber view at baseline (white) and follow-up (black). All non-significative changes from baseline to follow-up with exception of AS segment; * $\mathrm{p}=0.009 ; \mathrm{AS}=$ Antero-septal, IS=Infero-septal, $\mathrm{I}=$ Inferior, $\mathrm{IL}=$ Infero-lateral, $\mathrm{AL}=$ Antero-lateral, $\mathrm{A}=$ Anterior. BS=Basal-septal, MS=Medio-Septal, ApS=Apical-septal, ApL=Apical-lateral, $\mathrm{ML}=$ Medio-lateral, $\mathrm{BL}=\mathrm{Basal}-$ lateral.

Figure 4: Short-axis displacement curves: Specific behavior of a responder (top) versus a non-responder (bottom) patient from baseline (left) to follow-up (right) Top: Typical pattern of displacement from baseline to follow-up in a responder. (yellow curve: antero-septal, blue curve: infero-lateral). Observe the inversion and delay of septal onset of displacement (yellow arrow) and the inversion of peak displacement at follow-up. Bottom: Typical pattern of displacement from baseline to follow-up in a non-responder. (yellow curve: anteroseptal, blue curve: infero-lateral). Observe the absence of temporal inversion and delay of antero-septal onset of displacement (yellow arrow) and the absence of peak displacement inversion at follow-up. 


\section{Figure 1}

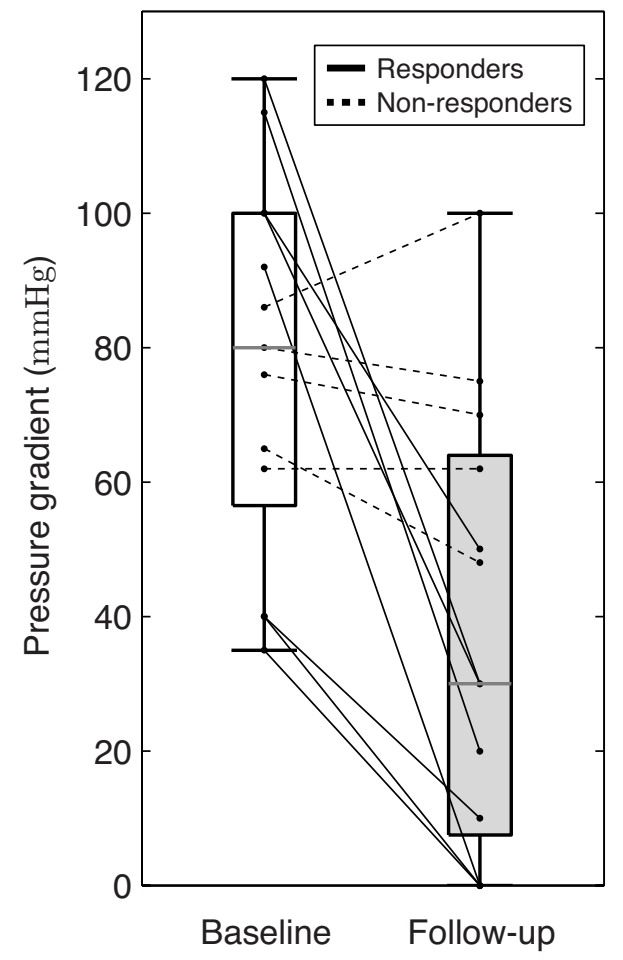

\section{Figure 2}
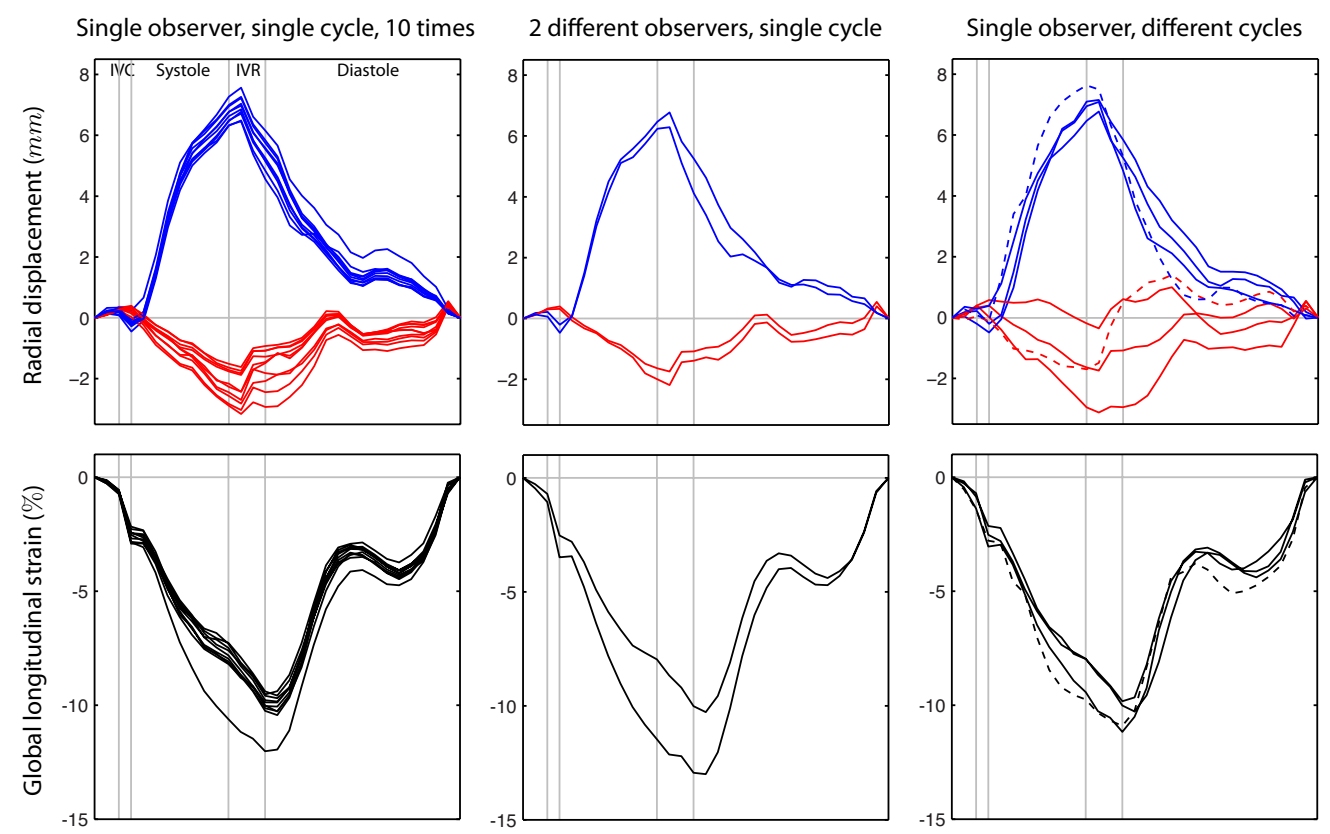

- Basal septum

- Basal lateral wall -.. Consecutive cycles 
Preprint version accepted to appear in International Journal of Cardiovascular Imaging.

Final version of this paper available at http://www.springerlink.com

Figure 3
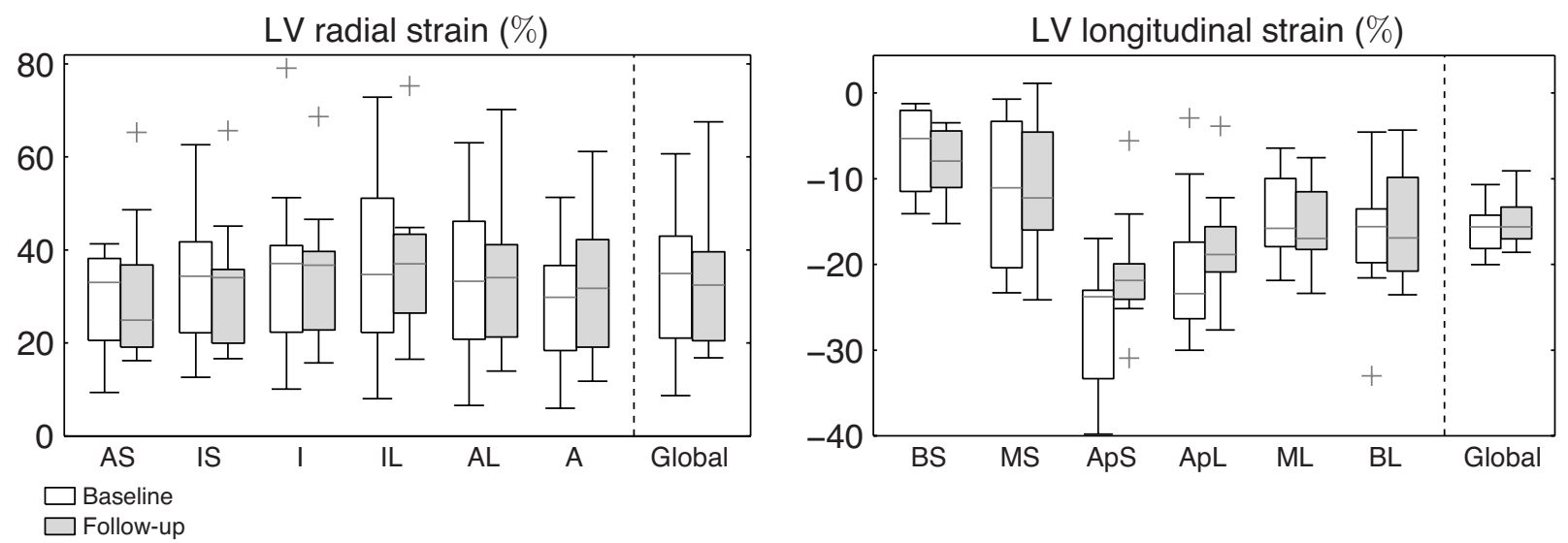

\section{Figure 4}
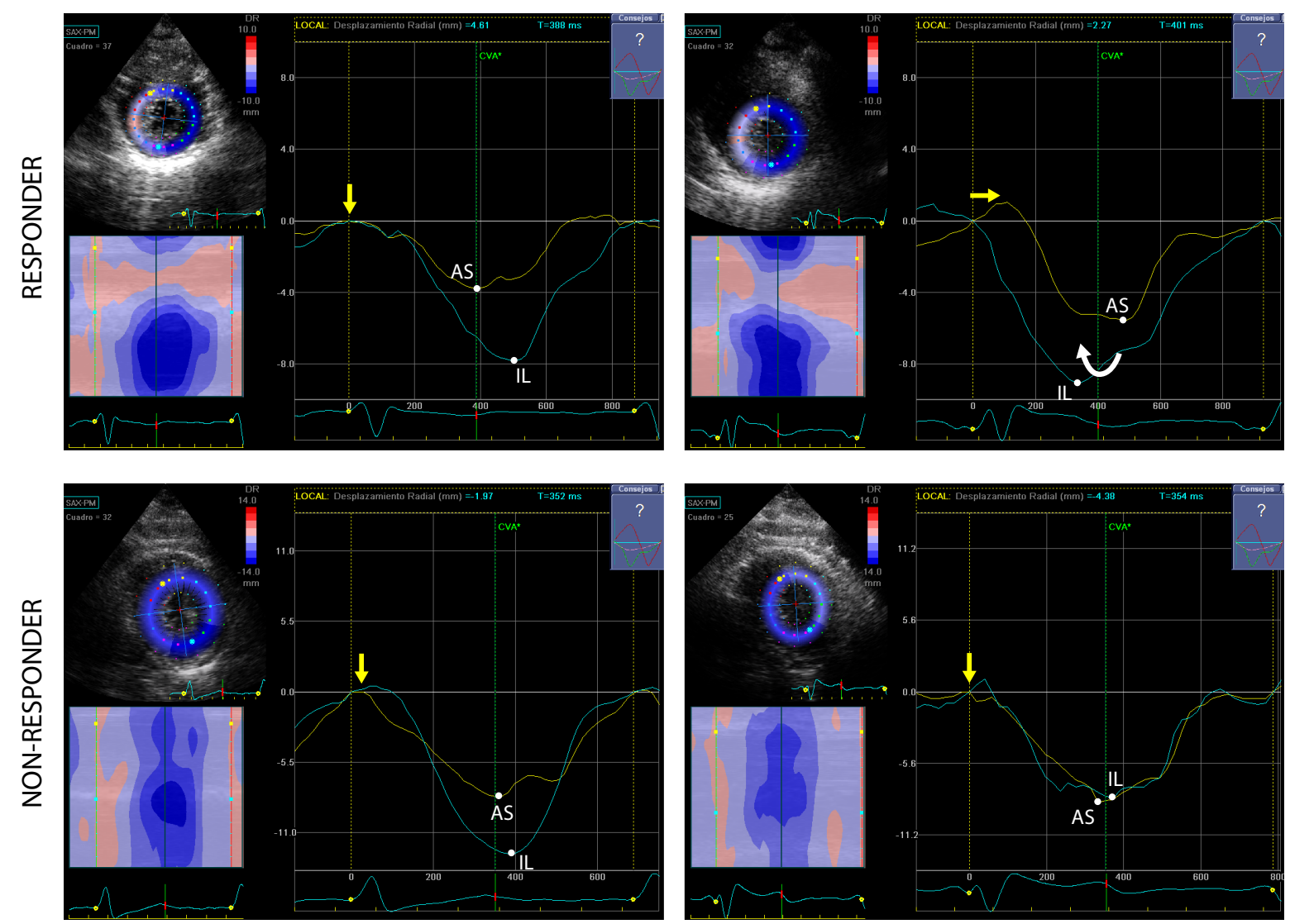

$\mathrm{IL}=$ Infero-Lateral

AS $=$ Antero-Septal 